\title{
Correction to Crowdsourcing to promote HIV testing among MSM in China: study protocol for a stepped wedge randomized controlled trial
}

\author{
SESH Study Group and Joseph D. Tucker
}

\section{Correction}

In the original publication [1] are errors in the acknowledgement section in the backmatter. The corrections can be found below in this Erratum.

Incorrect affiliation 4:

Guangdong Provincial Center for Skin Diseases and Sexually Transmitted Infections Control, No. 2 Lujing Road, Guangzhou, China, 510095.

Correct affiliation 4:

Guangdong Provincial Center for Skin Diseases and Sexually Transmitted Infections Control Center, Dermatology Hospital, Southern Medical University, No. 2 Lujing Road, Guangzhou, China, 510095.

Incorrect author affiliation:

Meizhen Liao ${ }^{15}$, Dianmin Kang ${ }^{15}$

Correct author affiliation:

Meizhen Liao ${ }^{16}$, Dianmin Kang ${ }^{16}$

Missing affiliation 16:

Shandong Provincial Center for Disease Control and Prevention, Jinan, China

Published online: 23 October 2017

\section{Reference}

1. Crowdsourcing to promote HIV testing among MSM in China: study protocol for a stepped wedge randomized controlled trial. Trials. 2017;18:447 10.1186/ s13063-017-2183-1. 